\title{
DESIGNER DRUGS: ASPECTOS ANALÍTICOS E BIOLÓGICOS
}

Rachel Bulcão, Solange Cristina Garcia*, Renata Pereira Limberger e Marília Baierle

Departamento de Análises, Faculdade de Farmácia, Universidade Federal do Rio Grande do Sul, Av. Ipiranga, 2752, 90610-000

Porto Alegre - RS, Brasil

\section{Marcelo Dutra Arbo}

Centro de Informação Toxicológica do Rio Grande do Sul, Fundação Estadual de Produção e Pesquisa em Saúde, Rua Domingos Crescêncio, 132, 90650-090 Porto Alegre - RS, Brasil

Alice Aparecida da Matta Chasin

Faculdades Oswaldo Cruz, Rua Brigadeiro Galvão, 540, 01151-000 São Paulo - SP, Brasil

Flávia Valladão Thiesen

Faculdade de Farmácia, Pontifícia Universidade Católica do Rio Grande do Sul, Av. Ipiranga, 6681, Prédio 12, 90619-900 Porto Alegre - RS, Brasil

\section{Rejane Tavares}

Departamento de Métodos Diagnósticos, Universidade Federal de Ciências da Saúde de Porto Alegre, Rua Sarmento Leite, 245, 90050-170 Porto Alegre - RS, Brasil

Recebido em 17/12/10; aceito em 12/7/11; publicado na web em 2/9/11

\begin{abstract}
DESIGNER DRUGS: ANALYTICAL AND BIOLOGICAL ASPECTS. In the recent years, analytical toxicologists have been facing difficulties in detecting designer drugs due to the chemical modifications on the existing structures and the speed in which they are released into the market, requiring the development and improvement of specific and appropriate analytical methods. This work is a review of the literature which summarizes the characteristics of the drugs and the analytical validated methods using conventional and unconventional matrices currently used for correct identification and quantification of the following classes of emerging drugs of abuse: derivatives of opiates, amphetamines, tryptamines, piperazines and cannabinoids.
\end{abstract}

Keywords: biological matrices; drug abuse; toxicology.

\section{INTRODUÇÃO}

As chamadas designer drugs (drogas planejadas, ou seja, análogos estruturais) estão entre as drogas de abuso mais utilizadas no Ocidente. Seus efeitos psicotrópicos específicos, que fundamentam sua utilização como drogas de abuso, são descritos como capacidade aumentada da comunicabilidade, empatia e autoconhecimento, o que distingue esta classe de compostos das substâncias estimulantes e alucinógenas propriamente ditas, que produzem estados de euforia e agitação e, alucinações visuais e auditivas, respectivamente. ${ }^{1,2}$

A utilização de substâncias psicoativas é antiga na história da civilização, as primeiras experiências humanas ocorreram por meio do consumo de plantas. A partir do século XIX, o homem isolou princípios ativos vegetais como morfina, cocaína e efedrina. Porém, foi no final do século passado, com o surgimento das anfetaminas, que uma substância psicoativa foi totalmente sintetizada em laboratório. Com o aparecimento das drogas sintéticas, nos anos 80, ocorreu a popularização das designer drugs. Essas drogas têm como característica essencial o fato de terem sido modificadas em laboratório, com o intuito de potencializar ou criar efeitos psicoativos ou evitar efeitos indesejáveis, além de burlar a legislação vigente. Além disto, a disponibilidade e a diminuição do custo tecnológico permitem que tais drogas sejam sintetizadas com facilidade em laboratórios clandestinos domésticos. ${ }^{3-5}$

Nos anos 90, com a popularização da internet, houve uma enorme divulgação e distribuição das designer drugs. ${ }^{5,6}$ Entretanto, foi na primeira década do século XXI que o número de novos usuários de substâncias ilícitas aumentou de forma significativa, com relatos da apreensão de diversas designer drugs em todo o mundo. ${ }^{7,8}$

*e-mail: solange.garcia@ufrgs.br
As designer drugs mais utilizadas são os compostos anfetamínicos, tais como 3,4-metileno-dioxi-anfetamina (MDA), 3,4-metilenodioxi-metanfetamina (MDMA, ecstasy), $p$-metoxi-anfetamina (PMA) e $p$-metoxi-metanfetamina (PMMA). No Brasil, o uso recreacional tem sido constatado em vários pacientes que buscam tratamento nas clínicas de reabilitação, além de apreensões destas drogas, sendo, portanto, de grande relevância sua correta identificação e/ou quantificação para fins clínicos e forenses. ${ }^{9}$

Do ponto de vista analítico, pode-se considerar um grande desafio a correta identificação desta classe de drogas. A dificuldade em se analisar designer drugs por métodos estabelecidos é devida à versatilidade das modificações ocorridas nas moléculas e à velocidade com que elas surgem no mercado, sendo mais rápido o desenvolvimento de novas moléculas do que de métodos adequados para identificação inequívoca e, portanto, diagnóstico de uso. Diferentemente das drogas clássicas, a maioria destes compostos não possui metodologias específicas para realização de triagens utilizadas na rotina laboratorial. Mesmo quando se utilizam métodos analíticos mais abrangentes, incluindo a espectrometria de massas (EM), estas novas drogas podem não ser detectadas, ou devido aos espectros de massas não estarem presentes nas bibliotecas espectrais de referência, ou porque os métodos estabelecidos, utilizando monitoramento seletivo de íons (MSI) enfocando os fragmentos das drogas clássicas, geralmente não incluem fragmentos típicos das novas designer drugs. ${ }^{10}$ Portanto, para acompanhar a evolução do mercado de drogas ilícitas é necessário adaptar continuamente os métodos de análise existentes ou desenvolver novos métodos que permitam a determinação correta destes novos compostos, assim como a aquisição de padrões de referência destes novos compostos.

Dessa forma, o objetivo deste trabalho foi realizar uma revisão da literatura a respeito dos métodos validados, bem como das matrizes 
biológicas convencionais e alternativas atualmente utilizadas para a correta identificação de designer drugs.

\section{ASPECTOS BIOLÓGICOS E ANALÍTICOS DAS DESIGNER DRUGS}

As designer drugs são produzidas baseadas na premissa de ressintetizar drogas já existentes, a fim de que se obtenham os mesmos efeitos psicoativos com moléculas quimicamente diferentes da droga original. Como a estrutura molecular da droga é alterada, estas não são definidas como proscritas, burlando a legislação vigente..$^{9-12}$

As primeiras designer drugs que surgiram eram narcóticas derivadas da heroína. ${ }^{13,14}$ Depois surgiram os derivados das feniletilaminas que incluem a 2-metil-amino-3,4-metileno-dioxi-propiofenona (metilona), N-metil-1-(1,3-benzodioxol-5-il)-2-butanamina (MBDB) e a 2,5-dimetoxi-4-iodo-feniletilamina (2C-I) ${ }^{15}$ Alguns derivados da triptamina incluem $\alpha$-metil-triptamina (AMT), 5-metoxi- $\alpha$-metil-triptamina (5-MeO-AMT), 5-metoxi-N,N-di-isopropil-triptamina (5-MeO-DIPT), e 5-metoxi-N,N-metil-isopropil-triptamina (5-MeO-MIPT). Mais recentemente surgiram as designer drugs derivadas da piperazina, sendo as mais utilizadas a 1-(3-clorofenil)piperazina ( $m \mathrm{CPP})$, a 1-benzilpiperazina (BZP), a 1-(4-metoxifenil)-piperazina (MeOPP) e a 1-(3-trifluorometilfenil)-piperazina (TFMPP), ${ }^{15-17}$ e os derivados canabinoides como canabicicloexanol, 1-pentil-3-(1-naftoil)indol (JWH-018), 1-naftoil(1-butilindolil)metanona (JWH-073) e 2,3-hidroxicicloexil-5-(2-metiloctanil)-fenol (CP 47,497). ${ }^{18}$

O mais popular, MDMA, foi desenvolvido como designer drug na década de $70,{ }^{19}$ mas é proscrito em muitos países devido aos efeitos tóxicos, ${ }^{19,20}$ destacando-se o comprometimento dos axônios no encéfalo, principalmente no córtex e outras áreas cerebrais superiores. ${ }^{19}$ Outras regiões cerebrais também afetadas incluem as áreas hipotalâmicas, que ajudam na regulação da temperatura, no comportamento alimentar e ritmos biológicos. ${ }^{19} \mathrm{O}$ MDMA possui menos efeitos sobre os sistemas dopaminérgico e noradrenérgico do que outras metanfetaminas, atuando principalmente sobre a neurotransmissão serotonérgica. ${ }^{2}$ Derivados do MDMA, como metilona, MBDB e benzodioxolilbutanamina (BDB), estão agora disponíveis no mercado ilícito e são utilizados para obter os mesmos efeitos que o MDMA. ${ }^{21}$

\section{Derivados opioides}

Os mais utilizados são os derivados da fentanila (Figura 1) que, no final dos anos 70, foram desenvolvidos e comercializados como um substituto da heroína, com capacidade semelhante de bloqueio da dor e de causar euforia. Alguns compostos do grupo fentanila, como alfentanila, sufentanila e remifentanila, são usados como anestésicos e analgésicos de alta potência e curta duração de ação. ${ }^{3,22,23}$

De forma geral, derivados da fentanila são produzidos a baixo custo e comumente usados de maneira abusiva. Em vista disso, no final da década de 80 , houve aumento no número de relatos sobre a produção ilegal, apreensão e uso ilícito desta classe de substâncias.

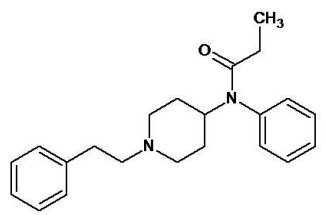

Fentanila

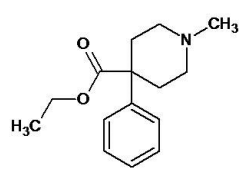

Meperidina
Figura 1. Estruturas químicas da fentanila e meperidina, protótipos das designer drugs derivadas de opioides
Devido à alta potência são utilizados em doses baixas concomitantemente com outros compostos, ocasionando overdoses acidentais. ${ }^{24}$

Outro grupo de derivados opioides são os derivados da meperidina (Figura 1), conhecida pelo nome comercial de Demerol ${ }^{\circledR}$, com uso medicinal reconhecido, porém apresentando um alto potencial de abuso. Um dos derivados da meperidina, a 1-metil-4-fenil-4-propionpiperidina (MPPP) é popular entre os consumidores de droga porque, quando injetada, produz euforia semelhante à heroína. Em alguns casos, pode conter como impureza 1-metil-4-fenil-1,2,5,6-tetra-hidropiridina (MPTP), identificada no ínicio dos anos 80, que causa danos cerebrais e pode resultar em uma síndrome idêntica à doença de Parkinson. A partir de uma única dose desta substância, pode ser observado aumento do tônus muscular, dificuldade de movimentos e fala, salivação e rigidez das extremidades superiores. ${ }^{23,25,26}$

Alguns derivados opioides são encontrados no material biológico em concentrações muito baixas, e nestas concentrações não podem ser detectados por meio de triagem com procedimentos rotineiramente empregados. Portanto, métodos diretos de determinação deste grupo de compostos são necessários. Um método utilizando cromatografia líquida acoplada a um espectrômetro de massas (CLAE/EM) foi usado para a determinação de fentanila em casos forenses. ${ }^{27}$ Foi possível detectar e quantificar fentanila em amostras de sangue, bile e do pulmão colhidas durante a necropsia de pessoas que morreram logo após procedimento cirúrgico em que a fentanila foi administrada como um adjuvante para a anestesia geral. ${ }^{28} \mathrm{~A}$ análise da urina dos toxicodependentes foi realizada por cromatografia gasosa de alta resolução acoplada a um espectrômetro de massas (CG/EM) para detectar os metabólitos e os compostos inalterados, a fim de comprovar o abuso. ${ }^{23,27-29}$

\section{Derivados anfetamínicos}

A classe da anfetamina e derivados anfetamínicos inclui todas as substâncias com uma estrutura de feniletilamina substituída. $\mathrm{Na}$ Figura 2, encontram-se as estruturas químicas dos principais derivados das feniletilaminas. Os anfetamínicos com substituição no anel têm sido os mais encontrados no mercado ilícito e a maioria destes derivados é proveniente de laboratórios clandestinos. ${ }^{30}$ Apesar de algumas substâncias estarem incluídas na relação de drogas ilícitas da Convenção Internacional sobre Psicotrópicos desde 1977 como, por exemplo, o 2,5-dimetoxi- $\alpha, 4$-dimetil-feniletilamina (DOM), a maioria foi incluída na lista de 1986. Estes compostos diferem apenas

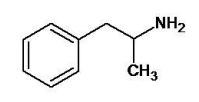

Anfetamina

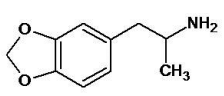

MDA
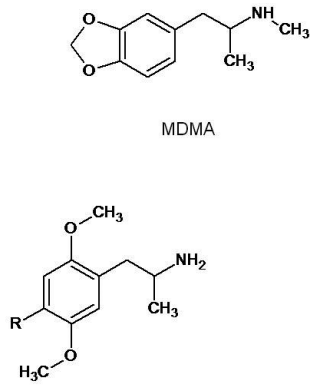

$\mathrm{R}=\mathrm{Br} ; \mathrm{DOB}$ $\mathrm{R}=\mathrm{I}$; $\mathrm{DOI}$ $\mathrm{R}=\mathrm{CH}_{3} ; \mathrm{DOM}$ $\mathrm{R}=\mathrm{CH}_{2} \mathrm{CH}_{3} ; \mathrm{DOET}$
$\mathrm{R}=\mathrm{CH}_{2} \mathrm{CH}_{2} \mathrm{CH}_{3} ; \mathrm{DOPR}$
Figura 2. Estruturas químicas da anfetamina e dos seguintes derivados anfetamínicos: 3,4-metileno-dioxi-anfetamina (MDA), 3,4-metileno-dioximetanfetamina (MDMA), 3,4-metileno-dioxi-etil-anfetamina (MDEA), 2,5-dimetoxi-4-bromo-anfetamina (DOB), 2,5-dimetoxi-4-iodo-anfetamina (DOI), 2,5-dimetoxi- $\alpha$,4-dimetil-feniletilamina (DOM), 2,5-dimetoxi-4-etilanfetamina (DOET), 2,5-dimetoxi-4-propil-anfetamina (DOPR) 
na velocidade e duração da ação, potência e capacidade de modificar o ânimo, produzindo, ou não, alucinações. ${ }^{30,31}$

As metanfetaminas alcançaram maior popularidade na Costa Leste dos EUA e nos países orientais. Foram as primeiras designer drugs derivadas das feniletilaminas sintetizadas no Japão, em 1919. Podem ser administradas por via oral ou injetável, podendo ser ainda inaladas e fumadas. São lipossolúveis, atravessam facilmente a barreira hematoencefálica e concentram-se especialmente nos rins, pulmões, líquido céfalorraquidiano e cérebro. ${ }^{32}$ Além de riscos agudos, tais como a overdose, o uso crônico pode provocar forte dependência, podendo surgir alucinações e delírios, sintomas denominados "psicose anfetamínica", além de haver evidências de neurotoxicidade destas substâncias. ${ }^{33}$

A PMA, a PMMA e a 4-fluoroanfetamina (4-MTA) são classificados como os novos "clássicos" anfetamínicos. A PMA e a PMMA são anfetamínicos modificados, de forma a tornar mais intenso os efeitos centrais. Estas substâncias têm sido vendidas como ecstasy, sob a forma de comprimidos, e diversas mortes por overdose já foram relatadas, preocupando órgãos governamentais. ${ }^{34,35}$

A 4-MTA atua de forma semelhante às metanfetaminas, porém, este é um anfetamínico modificado, menos potente que os derivados convencionais. Foi desenvolvido por Dave Nichols (Purdue University) nos anos 70. A ocorrência de mortalidade relacionada à 4-MTA, provavelmente por sua afinidade serotoninérgica, desencorajou novos estudos. A presença da substância já foi detectada na Holanda, Inglaterra, Alemanha e Portugal. ${ }^{36}$

As metoxifeniletilaminas, como a 4 -iodo-2,5-dimetoxi- $\beta$ feniletilamina (2C-I), a 4-etil-2,5-dimetoxi- $\beta$-feniletilamina (2C-E), a 4-bromo-2,5-dimetoxi- $\beta$-feniletilamina (2C-B), a 4-cloro-2,5dimetoxi- $\beta$-feniletilamina (2C-C), a 3,4,5-trimetoxi-anfetamina (TMA), a 2,4,5-trimetoxi-anfetamina (TMA-2) e a 2,3,5-trimetoxi-anfetamina (TMA-4), exercem efeitos menos pronunciados sobre a recaptação e liberação de monoaminas na transmissão pré-sináptica, mas podem ser mais acentuadas nos efeitos pós-sinápticos. ${ }^{15}$

Uma dessas novas classes de drogas de abuso são as chamadas 2Cs, já citadas anteriormente: 2C-B, 2C-I, 2C-C, 2C-E, a 4-etiltio-2,5-dimetoxi- $\beta$-feniletilamina $(2 \mathrm{C}$-T-2) e a 2,5 -dimetoxi-4propiltio- $\beta$-feniletilamina ( $2 \mathrm{C}-\mathrm{T}-7)$. Suas estruturas químicas são mostradas na Figura $3 .{ }^{37} \mathrm{O} 2 \mathrm{C}-\mathrm{B}$, juntamente com o $2 \mathrm{C}-\mathrm{T}-7$ são psicodélicos que foram desenvolvidos por Alexander Shulgins durante os anos 70, enquanto explorava variedades de 2,5-dimetoxi-4-bromo-anfetamina (DOB). Seus efeitos são bastante semelhantes aos do ácido lisérgico (LSD), no entanto, os efeitos colaterais gastrointestinais (náuseas, vômitos, diarréia) e sintomas de pânico e confusão mental são recorrentes e podem expor os usuários a<smiles>COc1cc(CN)c(OC)cc1Br</smiles>

$2 \mathrm{C}-\mathrm{B}$<smiles>COc1cc(CN)c(OC)cc1I</smiles>

$2 \mathrm{C}-\mathrm{I}$
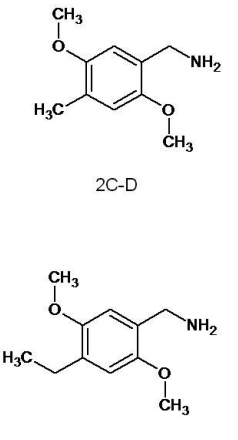

$2 \mathrm{C}-\mathrm{E}$
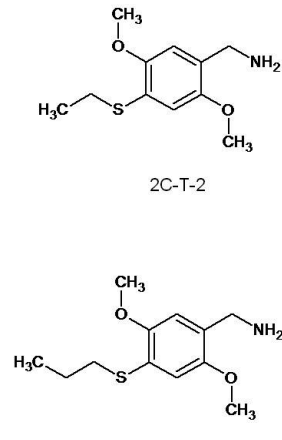

$2 \mathrm{C}-\mathrm{T}-7$
Figura 3. Estruturas químicas dos derivados 2Cs: 2,5-dimetoxi-4-bromofeniletilamina (2C-B), 2,5-dimetoxi-4-metil-feniletilamina (2C-D), 4-etil-tio2,5-dimetoxi- $\beta$-feniletilamina (2C-T-2), 2,5-dimetoxi-4-iodo-feniletilamina (2C-I), 2,5-dimetoxi-4-etil-feniletilamina $(2 C$-E) e 2,5-dimetoxi-4-propiltio$\beta$-feniletilamina $(2 C-T-7)$ situações de risco. ${ }^{38,39}$ Em 1985, as 2Cs ganharam popularidade nos EUA e Europa quando o MDMA se tornou ilegal. Em 1999, elas tornaram-se ilegais na maior parte do mundo, porém em alguns países a $2 \mathrm{C}-\mathrm{B}$ continua a ser vendida ilegalmente como substituta do ecstasy. ${ }^{38,40,41} \mathrm{O}$ significado de sua abreviação (2C-B) é devido à presença de uma cadeia com dois átomos de carbono ligados a um anel (2C), bem como a um átomo de bromo. Classificada como alucinógeno, juntamente com o MDMA e o LSD, porém mais potente que o MDMA (dez vezes), ${ }^{42}$ e menos que o LSD, a 2C-B combina a ação estimulante do tipo anfetamina e a ação alucinógena do tipo mescalina. ${ }^{40,41}$

Utilizando os resultados de algumas análises de designer dru$g s$, é possível fazer previsões da potência alucinógena das novas $\beta$-feniletilaminas. Para algumas 2 Cs estão disponíveis métodos para screening e para quantificação em amostras de plasma e urina, ${ }^{43-45}$ sendo que as técnicas analíticas empregadas são CG/EM, cromatografia gasosa com detector de nitrogênio e fósforo (CG/ $\mathrm{DNF})^{46}$ e eletroforese capilar acoplada à espectrometria de massas (EC/EM). ${ }^{47,48}$

Por outro lado, em 2004, foi apresentado um método utilizando a CG/EM para a detecção de compostos $2 \mathrm{C}$ na urina. No entanto, para a análise toxicológica, principalmente na urina, é importante conhecer previamente as reações de biotransformação dos compostos, especialmente se eles são excretados inalterados ou na forma de metabólitos. ${ }^{49}$ Além disso, os dados sobre a toxicocinética são necessários para a avaliação de riscos toxicológicos, pois os metabólitos podem desempenhar um papel importante na toxicidade de um fármaco e/ ou outro xenobiótico. Alguns estudos têm sido publicados sobre o processo de biotransformação de substâncias psicoativas, principalmente das feniletilaminas. ${ }^{50,51}$ Para a $2 \mathrm{C}-\mathrm{B}$, estudos investigaram o processo da biotransformação, procurando identificá-la qualitativa e quantitativamente em hepatócitos de $\operatorname{ratos}^{52}$ e metabólitos na urina. ${ }^{5,51,53,54}$ Foi publicado, também, o processo de biotransformação hepático da 2C-B em seis diferentes espécies, incluindo humanos e camundongos. ${ }^{41}$ Para a 2C-T-2, foram realizados estudos preliminares em urina de humanos. ${ }^{53}$

No que diz respeito aos alucinógenos derivados das feniletilaminas, a mescalina, encontrada no cactus peiote, ${ }^{55}$ é usada como padrão contra o qual todas as outras feniletilaminas são comparadas. ${ }^{56} \mathrm{~A}$ TMA foi o primeiro psicodélico alucinógeno totalmente sintético, estruturalmente relacionado com a mescalina. A TMA-2 é outro derivado sintético e um dos isômeros da TMA, porém mais potente do que a TMA e a mescalina. ${ }^{55,57}$ A TMA e alguns de seus isômeros estão representados na Figura 4.
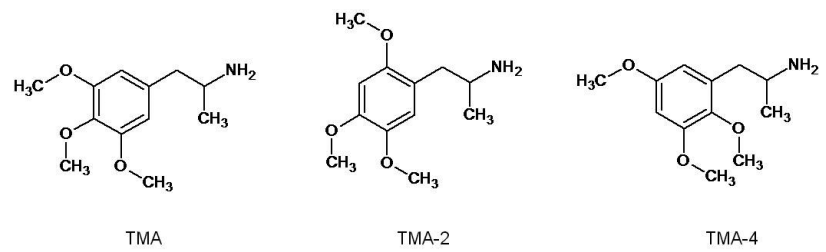

Figura 4. Estruturas químicas das feniletilaminas 3,4,5-trimetoxi-anfetamina (TMA), 2,4,5-trimetoxi-anfetamina (TMA-2) e 2,3,5-trimetoxi-anfetamina (TMA-4)

\section{Derivados da triptamina}

A estrutura geral das triptaminas encontra-se na Figura 5. A semelhança dos seus efeitos psicofarmacológicos e sua capacidade de produzir tolerância cruzada indicam que as indolaminas, como os derivados da triptamina e os alucinógenos fenilamínicos, possuem mecanismos semelhantes. Diferentemente das feniletilaminas, que 
são agonistas seletivos $5-\mathrm{HT}_{2}$, as indolaminas são agonistas 5-HT não seletivos. ${ }^{58}$

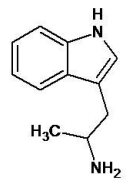

AMT

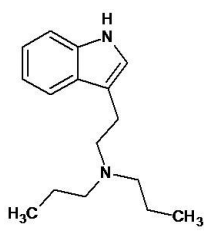

DPT

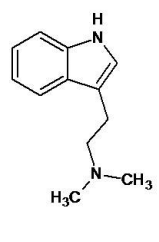

DMT

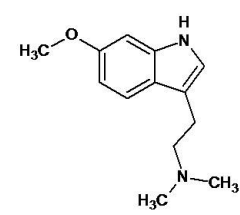

5MeO-DMT
Figura 5. Estruturas químicas gerais dos derivados da triptamina: $\alpha$-metiltriptamina (AMT), dimetil-triptamina (DMT), N,N-dipropil-triptamina (DPT), 5-metoxi-N,N-dimetil-triptamina (5-MeO-DMT)

Os derivados da triptamina, principalmente a 5-metoxi- $\alpha$-metiltriptamina (5-MeO-AMT), inibem fortemente a recaptação e aumentam a liberação das monoaminas. Já a N,N-dipropil-triptamina (DPT), a 5-MeO-DIPT, a 5-MeO-MIPT e a 5-metoxi-N,N-dimetil-triptamina (5-MeO-DMT) inibem apenas a recaptação das monoaminas. ${ }^{16}$

A N,N-dimetil-triptamina (DMT) é um composto tipicamente psicoativo (agonista serotoninérgico) derivado da triptamina que existe em milhares de espécies de plantas. Existem compostos estruturalmente relacionados, como a 5-MeO-DMT, bufotenina, psilocibina e psilocina, os quais são também produtos naturais. ${ }^{55,59}$

A Ayahuasca, uma mistura sul-americana de plantas, é preparada a partir de duas espécies vegetais contendo alcaloides de núcleo harmano (da Banisteriopsis caapi) e DMT (da Psychotria viridis). ${ }^{60}$ Esta combinação é importante porque a DMT, presente na Ayahuasca, é metabolizada rapidamente no organismo pela monoamina oxidase (MAO) e, por isso, não é psicoativa quando usada oralmente a menos que combinada com algum inibidor da $\mathrm{MAO},{ }^{55}$ como os alcaloides harmina e harmalina, que contêm o núcleo triptamina.

A 5-MeO-DIPT também produz efeitos farmacológicos semelhantes aos da DMT, porém é um alucinógeno ativo por via oral. ${ }^{61,62}$

Em 2005, estas substâncias foram investigadas através de técnicas analíticas simultâneas, ${ }^{55}$ utilizando CG/EM e CLAE/ESI-EM (cromatografia líquida com ionização por electrospray) para determinação de triptaminas e feniletilaminas não controladas e para diferentes tipos de triptaminas e feniletilaminas controladas, que são originalmente encontradas em fungos ou plantas. Além disso, a análise destas drogas foi realizada em diferentes tipos de produtos, que foram anunciados como potencialmente psicotrópicos. Como resultado das análises, a 5-MeO-DIPT foi encontrada em 8 de um total de 99 tipos de produtos. Além disso, DMT, harmina e harmalina (a partir de plantas secas) também foram encontradas em alguns dos 99 produtos. Dessa forma, estes métodos analíticos poderiam ser úteis também para o estudo da distribuição das triptaminas e feniletilaminas não controladas. ${ }^{55}$

Em 2008, Takahashi e colaboradores analisaram diferentes derivados de feniletilaminas e triptaminas presentes nas designer drugs utilizando CG acoplada a um espectrômetro de massas, e ressonância magnética nuclear (RMN). Devido à dificuldade em se conseguir padrão destas substâncias, eles utilizaram compostos sintetizados, isolados e purificados. Concluíram que a CG/EM era sensível o suficiente para a determinação destes compostos, na faixa de microgramas. ${ }^{63}$

\section{Derivados da piperazina}

A piperazina e alguns derivados têm uso médico com agentes anti-helmínticos. ${ }^{64}$ Os principais derivados da piperazina são apresentados na Figura 6. A benzilpiperazina (BZP) foi testada na década de 70 como uma possível droga para produção de medicamento antidepressivo, entretanto, por ter apresentado propriedades semelhantes à anfetamina em testes com animais e humanos, o estudo foi interrompido. ${ }^{65,66}$ Embora seu uso terapêutico não tenha sido liberado, existem relatos do seu uso como entorpecente, sendo identificada em uma necropsia forense, em 1999, ${ }^{64}$ e detectada em materiais encaminhados para análise e identificação de tóxico-entorpecentes. ${ }^{67}$

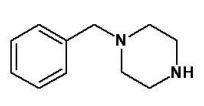

BZP<smiles>COc1ccc(N2CCNCC2)cc1</smiles>

MeOPP

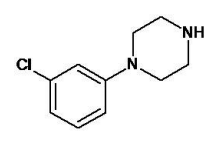

$m \mathrm{CPP}$

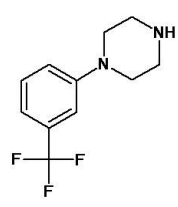

TFMPP
Figura 6. Estruturas químicas das piperazinas: benzilpiperazina (BZP), $m$ 1-(3-clorofenil) piperazina (mCPP), 1-(4-metoxifenil) piperazina (MeOPP) e 1-(3-trifluorometilfenil) piperazina (TFMPP)

A $m$ CPP é uma substância sintética derivada da piperazina e tem sido detectada em comprimidos de drogas estimulantes sintéticas, juntamente ou às vezes substituindo o MDMA, em comprimidos comercializados como ecstasy, além de adulterante no pó de cocaína, em mistura com anfetaminas e outros medicamentos, como a metoclopramida. ${ }^{6,68}$

Em 2006, foi apresentado um método analítico utilizando CLAE para a análise de materiais suspeitos de conterem a substância $m \mathrm{CPP}$, tais como comprimidos e amostras em pó apreendidas na Polônia. ${ }^{68}$

Em 2008, foram recebidos pelo Laboratório de Toxicologia Forense do Núcleo de Perícias Criminalísticas de Campinas - Superintêndencia da Polícia Técnico-Científca do Estado de São Paulo, comprimidos de diferentes cores, tamanhos e formatos, oriundos de apreensões realizadas em Campinas e cidades adjacentes para exame pericial. Os exames químico-periciais realizados apresentaram resultados inesperados, diferentes daqueles obtidos quando a substância analisada é a MDMA, que é principal droga de abuso consumida na forma de comprimidos no Brasil. A partir daí, foi iniciada uma investigação química para verificar a substância ativa presente nos comprimidos. Para isso, foi realizada uma triagem, com métodos colorimétricos, como o Teste de Marquis, comumente utilizado para triagem de compostos anfetamínicos, tais como MDMA. O material examinado permaneceu incolor, semelhante à cor apresentada pela cocaína, porém o resultado foi diferente dos obtidos pelos padrões de anfetamina (laranja) e MDMA (preto). No Teste de Scott, para detecção de cocaína e outros anestésicos locais, observou-se o aparecimento de coloração azul na placa de toque. Este resultado diferiu daquele observado para a MDMA, que apresentou solução rósea com precipitados de coloração azulada e o Teste de Simons, resultando 
em uma coloração laranja (até 30 s) e marrom escuro (após 2 min). Além disto, foi realizada uma triagem por cromatografia em camada delgada de alta eficiência (CCDAE) utilizando três diferentes sistemas solvente, seguida por cromatografia líquida com detector de arranjo de diodos (CLAE/DAD) e confirmação por espectrometria de massas (EM), confirmando a presença de CPP. ${ }^{69}$ Porém, a espectrometria de massas não distingue os isômeros $m \mathrm{CPP}, o \mathrm{CPP}$ e $p \mathrm{CPP}$, que são comumente encontrados em produtos apreendidos. ${ }^{70}$ Contudo, para fins legais, a diferenciação isomérica não foi necessária, pois a RDC n.79/2008 menciona no adendo 1.1 da lista F2 que devem ser controlados todos os sais e isômeros das substâncias elencadas na referida lista. ${ }^{71}$

As técnicas utilizadas para identificação e quantificação de BZP em fluidos biológicos geralmente são CG/EM e CLAE/EM. ${ }^{70}$

\section{Derivados canabinoides}

Após a descoberta dos receptores canabinoides $\mathrm{CB}_{1}$ (central) e $\mathrm{CB}_{2}$ (periférico), na década de 80 , assim como do sistema canabinoide endógeno e do seu papel fisiológico, um número de análogos canabinoides foi sintetizado e foram estudadas as suas atividades farmacológicas para o tratamento de diversas doenças. ${ }^{72}$ Esses compostos possuem estruturas químicas variadas, que vão desde derivados clássicos, que apresentam o anel dibenzopirano característico até estruturas não clássicas, derivadas do indol, indeno, pirrol, pirazol e cicloexanol, porém compartilhando a alta afinidade pelos receptores canabinoides. ${ }^{18}$ As estruturas químicas de alguns derivados com ação canabinomimética estão representadas na Figura 7. Sua ação sobre a saúde humana ainda não foi relatada, porém recidivas em pacientes que faziam uso prévio de Cannabis, resultando em episódios psicóticos, já foram relatadas. ${ }^{73}$

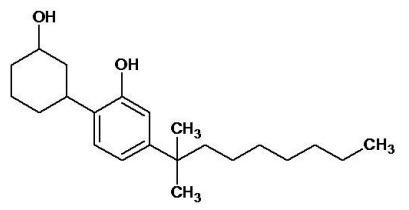

Canabicicloexanol

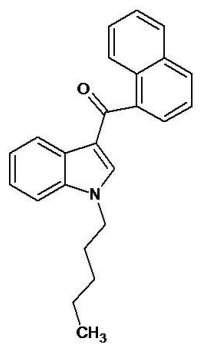

JWH-018
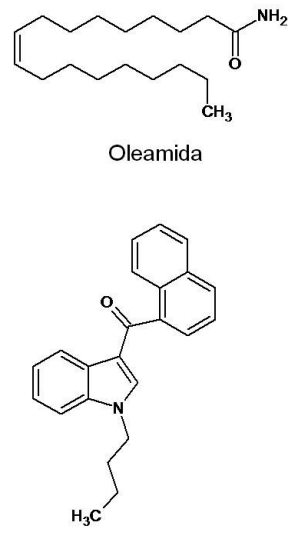

JWH-073
Figura 7. Estruturas químicas dos derivados canabinoides canabicicloexanol, JWH-018 (1-naftalenil(1-pentilindol-3-il)metanona) e JWH-073 (1-naftalenil(1-butil-1H-indol-3-il)metanona) e do canabinoide endógeno oleamida

Existem, até agora, poucos relatos sobre a toxicologia analítica dos derivados canabinoides. Os derivados canabicicloexanol, JWH018, JWH-073 e (1RS,3SR)-3-[4-(1,1-dimetiloctil)-2-hidroxifenil] cicloexan-1-ol foram detectados em produtos vegetais, comprados no Japão pela internet, após extração com metanol e ultrassonicação. Para a detecção foram utilizadas a cromatografia líquida de ultra eficiência acoplada ao espectrômetro de massas (CLUE/ESI-EM) e detector de arranjo de diodos (CLUE/DAD), além de CG/EM. ${ }^{18,72}$
Alguns derivados canabinoides foram analisados no sangue, utilizando CG/EM após extração em fase sólida e silanização. ${ }^{74}$ Foi conduzido um estudo para a detecção de metabólitos de JWH-018 na urina, em que os metabólitos se conjugaram com ácido glicurônico e foram detectados por CG e CLAE/EM/EM. ${ }^{75}$

\section{MÉTODOS ANALÍTICOS}

$\mathrm{Na}$ área forense, as análises toxicológicas são empregadas para fins de orientação de procedimentos judiciais, sendo desenvolvidas com a finalidade de estabelecer nexo causal entre o agente químico (toxicante) e a morte ou dano infligido ao homem. ${ }^{33}$ Para um diagnóstico toxicológico confiável é necessária a realização de uma análise toxicológica eficiente. Primeiramente é realizada uma triagem, por métodos gerais, principalmente quando não se conhece o agente tóxico a pesquisar. Estes métodos são empregados para verificar a ausência ou a presença de uma determinada classe ou grupo de substâncias. A importância da escolha de um método de triagem é fundamental, pois define a variedade de analitos que serão procurados e detectados. Portanto, deverá ter sensibilidade, eficiência e abrangência de compostos para ser considerado adequado. ${ }^{76}$ Do ponto de vista ético, resultados obtidos a partir de técnicas de triagem não podem ser considerados definitivos, devendo-se realizar métodos confirmatórios. ${ }^{77}$

Quase todas as designer drugs têm átomos de nitrogênio em suas estruturas moleculares. Assim, reagentes colorimétricos que reagem com nitrogênio são normalmente utilizados em testes de detecção, que incluem os reagentes de Marquis, Simon, Dragendorff, dentre outros. Ensaios contendo métodos analíticos baseados na espectroluminescência (fluorimetria, fosforimetria, quimioluminescência) são mais sensíveis do que os ensaios com detecção colorimétrica, porém exigem etapas de pré-tratamento da amostra. Um método sistemático preliminar para a seleção de designer drugs foi feito através da combinação de reagentes colorimétricos. No entanto, a sensibilidade foi menor do que a de técnicas cromatográficas, assim como a especificidade, necessitando purificação e condensação dos analitos presentes no material biológico. ${ }^{78}$

A EM é essencialmente indicada, principalmente em análises forenses, em que se recomenda também que, se o resultado analítico tiver uma importância na investigação criminal, o toxicante seja detectado em mais de uma matriz biológica. ${ }^{77}$

A identificação definitiva do tipo de droga geralmente requer uma análise aprofundada com a utilização de várias técnicas complementares, incluindo, além da EM, a espectroscopia no infravermelho (IV), difração de raios-x e RMN. ${ }^{79}$

Os métodos utilizados na análise de designer drugs em materiais biológicos podem ser compostos a partir de diferentes procedimentos de extração, separação, identificação e quantificação dos analitos presentes na amostra. A seleção da técnica de extração a ser usada, bem como o tipo de equipamento empregado na identificação e quantificação, deve levar em consideração além da finalidade a que se destina a análise e, portanto, da sensibilidade requerida, o tipo de equipamento disponível para a execução dos trabalhos. Em análises forenses, deve-se buscar sempre a elaboração de métodos sensíveis, seletivos e específicos, permitindo ao analista a emissão de resultados inquestionáveis e irrefutáveis., ${ }^{2,80,81}$

Além disto, a maioria dos testes rápidos para detecção de designer drugs, que são testes inespecíficos, é baseada em princípios imunológicos, podendo apresentar resultados falsos positivos ou falsos negativos. ${ }^{13,35,47,82-84} \mathrm{Em} 2004,{ }^{85}$ foi avaliada a interferência de determinadas substâncias estruturalmente semelhantes com alguma designer drug.

O pré-tratamento das amostras biológicas, que abrange as etapas de extração, pré-concentração e purificação, tem sido requerido nas 
Tabela 1. Principais técnicas analíticas e matrizes utilizadas na análise de designer drugs

\begin{tabular}{|c|c|c|c|}
\hline Designer drugs & Método analítico & Matriz biológica & Ref. \\
\hline MPPP, Fentanila & $\begin{array}{l}\text { CG/EM, CG/EM/EM, CLAE/EM, } \\
\text { CLAE/EM/EM, ELISA }\end{array}$ & $\begin{array}{l}\text { sangue, bile, pulmão, urina, cabelo, } \\
\text { soro }\end{array}$ & $23,27,28,29,111,112$ \\
\hline $\begin{array}{l}\text { 2C-T-7, 4-MTA, 2C-B, DOM, DOB, } \\
\text { 2C-I, PMMA, PMA, MDMA, MDEA, } \\
\text { MDA, MBDB, 2C-B, 2C-D, 2C-I }\end{array}$ & $\begin{array}{l}\text { CG/EM, EC/EM, CG-DNF, CLAE/ } \\
\text { EM, CG/EM/EM, CLAE/EM/EM, } \\
\text { CLAE-DF, ELISA }\end{array}$ & $\begin{array}{l}\text { sangue, urina, cabelo, saliva, plasma, } \\
\text { soro, humor vítreo, mecônio, suor }\end{array}$ & $\begin{array}{l}4,5,13,43,44,45,47,48,49,51, \\
55,63,83,112,114,115,116,120, \\
123,126,127,128,129\end{array}$ \\
\hline CPP, BZP, TFMPP & $\begin{array}{l}\text { métodos colorimétricos, CCDAE, } \\
\text { CLAE/DAD, CG/EM, CLAE/EM, } \\
\text { CLAE/EM/EM }\end{array}$ & $\begin{array}{l}\text { pós e comprimidos, urina, plasma, } \\
\text { sangue, soro }\end{array}$ & $13,64,67,68,69,70,130,131,132$ \\
\hline Triptaminas & $\begin{array}{l}\text { CG/EM, CLAE/EM/EM, CLAE/ESI- } \\
\text { EM, RMN }\end{array}$ & $\begin{array}{l}\text { produtos de origem vegetal, urina, } \\
\text { plasma, sangue, cabelo }\end{array}$ & $55,63,93,125$ \\
\hline Canabinoides & CLUE/DAD-ESI-EM e CG/EM & $\begin{array}{l}\text { produtos vegetais, urina, sangue, } \\
\text { cabelo, saliva }\end{array}$ & $18,72,74,75,113,133$ \\
\hline
\end{tabular}

*Definições das abreviações, ver texto.

análises cromatográficas de drogas para eliminar interferentes (compostos endógenos ou outras substâncias administradas concomitantemente com os analitos) e aumentar a sensibilidade e seletividade analíticas. ${ }^{86}$ As técnicas de extração líquido-líquido (ELL), extração em fase sólida (EFS) e microextração em fase sólida (MEFS) têm sido utilizadas em métodos convencionais de análises rotineiras e no preparo de amostras biológicas. ${ }^{86}$

Em análises realizadas por CG-MEFS, a fibra, após o processo de extração, é inserida no injetor aquecido e os analitos são rapidamente dessorvidos termicamente para a coluna cromatográfica; desta forma, as análises realizadas por CG-MEFS não utilizam solventes orgânicos e têm sido empregadas com êxito na extração de fármacos voláteis e semivoláteis em análises de amostras biológicas principalmente quando extraídos da fase gasosa, no modo headspace. Já os fármacos menos voláteis ou termicamente instáveis devem ser analisados após processos de derivatização/CGMEFS ou empregando-se a técnica MEFS acoplada, por exemplo, à cromatografia líquida de alta eficiência (MEFS-CLAE). ${ }^{86} \mathrm{Em}$ 1998, foi desenvolvido um método de triagem para 21 derivados anfetamínicos através da MEFS e CG/EM. ${ }^{83}$

Entre os diferentes métodos utilizados para a triagem de drogas em amostras biológicas, seja no âmbito do controle laboral, no controle rodoviário ou na generalidade dos casos forenses e consequente confirmação dos resultados positivos dessa triagem inicial, tem sido dada importância às técnicas de CG/EM, de CLAE/EM e aos imunoensaios, como a imunofluorescência polarizada (IFP). As principais técnicas analíticas, matrizes biológicas e designer drugs analisadas encontram-se resumidas na Tabela 1. Com o uso dessas técnicas temse procurado alcançar limites de detecção cada vez mais baixos e, ao mesmo tempo, ultrapassar algumas das limitações observadas com outros métodos como, por exemplo, a especificidade e a seletividade na identificação dos compostos.

Recentemente, Takahashi e colaboradores criaram uma biblioteca espectral para análise de 104 designer drugs através da determinação simultânea por CLAE/DAD e CG/EM e analisaram os dados obtidos. A partir destes dados foi possível realizar uma determinação rápida para confirmação destas substâncias, mesmo em caso de isômeros de posição, combinando informações do espectro ultravioleta, espectro de massas e tempos de retenção nos cromatogramas. ${ }^{63}$

\section{MATRIZES BIOLÓGICAS}

A análise química para a verificação do uso de drogas de abuso vem sendo requisitada com o objetivo de identificar o usuário, para que medidas de controle e prevenção sejam tomadas, já que esse uso está atingindo indivíduos de faixas etárias e camadas sociais variadas. Essas análises vêm sendo utilizadas por diversos segmentos da sociedade e aplicadas para verificar o uso de drogas no ambiente de trabalho, esporte, no auxílio e acompanhamento da recuperação de usuários em clínicas de tratamento e com finalidade forense. ${ }^{87}$

Cada matriz biológica possui vantagens e desvantagens. Conhecimentos sobre a estabilidade dos analitos no material biológico são de fundamental importância para garantir a confiabilidade dos resultados analíticos, uma vez que várias situações acabam por inserir intervalos de tempo variáveis entre a coleta do material, seu transporte até o laboratório e o momento da análise. ${ }^{9,76} \mathrm{~A}$ instabilidade dos analitos na matriz biológica e os fenômenos de redistribuição post mortem são interferentes importantes em toxicologia forense. ${ }^{76}$

A escolha do material biológico para realização de análises toxicológicas deve levar em consideração, principalmente, as características toxicocinéticas dos agentes investigados e a finalidade do diagnóstico laboratorial da intoxicação no auxílio/diagnóstico nos casos de envolvimento médico-legal. ${ }^{2}$ Após longos períodos, as análises ficam inviáveis devido à degradação sofrida pela matriz biológica, levando à formação de inúmeros interferentes que dificultam a identificação dos analitos de interesse frente aos demais produtos de decomposição presentes. ${ }^{88}$

Além disso, o aumento da sensibilidade nas técnicas analíticas tornou a detecção, a identificação e a quantificação de drogas em matrizes não convencionais mais práticas. Historicamente, a urina, o sangue, o humor vítreo e as amostras de tecidos foram considerados úteis. Porém, cabelos, unhas, saliva, estrato córneo, suor e outras matrizes biológicas têm sido utilizados como amostras não convencionais preferenciais, em alguns casos, para a análise de drogas, ou para complementar as análises realizadas em outras amostras. A tendência tem sido a de usar a análise de segmentos de cabelo para expandir a janela temporal do uso de drogas e fornecer informações sobre a cronologia de uso (Figura 8). Uma tendência mais recente é a de monitorar a secreção de drogas no suor pelo uso de patches. ${ }^{89,90}$

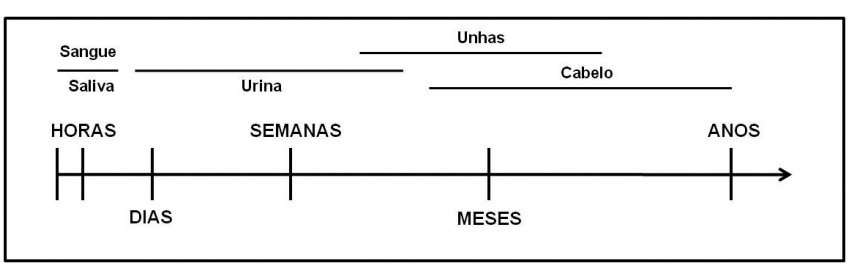

Figura 8. Tempo de detecção da maioria das drogas nas principais matrizes biológicas. Adaptada da ref. 134 


\section{Amostras convencionais}

\section{Sangue}

O sangue possui inúmeras vantagens, sendo uma das principais a possibilidade de correlacionar os níveis do xenobiótico encontrado com os efeitos desta substância. ${ }^{91}$ Esses achados, juntamente com dados sobre absorção, distribuição, biotransformação e excreção (toxicocinética) do xenobiótico, auxiliam na inferência sobre o momento de uso, quantidade de substância administrada e possíveis alterações fisiológicas e/ou psíquicas causadas pela substância. ${ }^{76}$

Em indivíduos vivos, os efeitos fisiológicos da maioria dos fármacos possuem correlação direta com suas concentrações no sangue e seus derivados (plasma e soro), fato que serve de base para a monitorização terapêutica de fármacos. Quando, entretanto, as amostras de sangue são coletadas post-mortem, são necessárias ressalvas, uma vez que fatores como o local de coleta e outros relacionados à redistribuição (cinética post-mortem) que eventualmente ocorreram quando cessados os fenômenos vitais podem modificar os valores encontrados. . $^{2,9,92}$

Em 2010, foi validado um screening para determinação de derivados anfetamínicos, triptamínicos e piperazínicos em amostras de soro por CLAE/EM/EM. Dentre os compostos encontram-se DMA, DOB, DOET, DOM, 4-MTA, PMA, PMMA, 3,4,5-TMA, TMA-6 e membros do grupo 2C: 2C-B, 2C-D, 2C-I, 2C-T-2, 2C-T-4 e 2CT-7. No grupo das triptaminas foram analisadas AMT, DPT, N,Ndiisopropil-triptamina (DiPT), DMT, 5-MeO-DMT, e BZP, MDBP, TFMPP, $m$ CPP e MeOPP no grupo das piperazinas. ${ }^{93}$

\section{Urina}

A urina, após o humor vítreo, que apresenta aproximadamente 98\% de água em sua constituição, é a matriz biológica com menor número de interferentes endógenos, pois é constituída principalmente por água e apresenta níveis significantes de proteína e lipídios (que poderiam interferir no processo de extração e identificação de fármacos) somente durante estados patológicos. Além disso, quando comparada ao sangue, frequentemente apresenta concentrações mais altas de xenobióticos e/ou de seus produtos de biotransformação. ${ }^{33}$

Contudo, os resultados estabelecem apenas que a droga de abuso foi administrada, uma vez que a correlação com os efeitos é baixa, devido à grande variedade de fatores que afetam a taxa de excreção de determinado composto e o volume urinário. ${ }^{94}$

\section{Conteúdo estomacal e tecidos}

O conteúdo estomacal constitui amostra de análise post-mortem, uma vez que prevalece no estômago a forma inalterada ${ }^{92} \mathrm{e}$ ionizada (aprisionamento farmacocinético) de algumas substâncias. Estas drogas podem estar presentes tanto na forma de resíduos da droga ingerida quanto pela possível secreção gástrica, desde que sejam bases fracas e passíveis de serem secretadas para o lúmem estomacal, onde se ionizam e permanecem até a posterior reabsorção no duodeno. ${ }^{4}$ Fragmentos de tecido muscular e vísceras como fígado, rins e cérebro também são materiais utilizados nos casos de necropsia. ${ }^{4,95,96}$ Porém, estas matrizes são referidas frequentemente na literatura. Este fato tem relação a estes materiais só estarem disponíveis nos casos de necropsia. $^{4}$

\section{Amostras não convencionais}

\section{Fluido oral}

A designação "fluido oral" representa a mistura de saliva (secreção de três glândulas principais: submandibular, parótida e sublingual) e outros constituintes presentes na boca, sendo composto por água, enzimas (principalmente amilase), glicoproteínas (mucina) e eletrólitos. Sua composição e o volume produzido inter- e intraindividual (cerca de 1 a $3 \mathrm{~mL} \mathrm{~min}^{-1}$ ) podem ser afetados por vários fatores como, por exemplo, ritmo circadiano, dieta, idade, doenças sistêmicas, como fibrose cística e diabetes mellitus, e utilização de algumas drogas e medicamentos, como os antidepressivos tricíclicos e outros anticolinérgicos, que levam à diminuição do volume de secreção bucal produzido. ${ }^{97}$

A saliva também se configura como amostra importante na determinação das designer drugs. A passagem de fármacos da corrente sanguínea para a saliva já havia sido sugerida desde a segunda metade da década de 60. Mesmo assim, a utilização de saliva como matriz biológica alternativa vem aumentando muito nos últimos anos, principalmente em estudos de farmacocinética, monitorização terapêutica e na verificação do uso de drogas ilícitas, particularmente por agências que monitoram motoristas quanto ao uso de substâncias psicoativas, dada a facilidade da coleta deste material na beira da estrada. ${ }^{2,98,99}$ A saliva possui ainda a vantagem de que sua coleta não é invasiva e provavelmente se constitui como o único fluido biológico através do qual se podem traçar paralelos com a concentração sanguínea, podendo ser empregada em estudos de farmacocinética e em análises forenses, por permitir correlacionar as concentrações obtidas com alterações comportamentais que possam eventualmente ser causadas pela droga. ${ }^{2,96,98}$

Para a análise deste tipo de material biológico, deve-se considerar que somente estão disponíveis para a difusão para a saliva aqueles compostos que não estiverem ligados a proteínas plasmáticas. A baixa taxa de ligação a proteínas (aproximadamente 20\%) de análogos das feniletilaminas, por exemplo, somada ao baixo peso molecular destes compostos, confere a estas substâncias passagem praticamente irrestrita entre o plasma e a saliva. ${ }^{2,100}$ Foram relatados usos de fluido oral para análises de derivados anfetamínicos utilizando EFS, seguido de CL/EM/EM, com analisadores de massas do tipo quadrupolo e tempo de voo ${ }^{100-102}$ utilizando CLAE com detector fluorescente (DF) ${ }^{103}$ e CG/EM. ${ }^{104,105}$

\section{Cabelo}

A análise do cabelo normalmente começa com uma triagem geral por imunoensaios, seguida de uma confirmação através de cromatografia. A CG/EM é a ferramenta analítica mais utilizada para determinação de drogas em cabelo. No entanto, a CLAE/EM é cada vez mais importante na determinação de drogas nesta matriz, devido à sua maior sensibilidade para compostos termolábeis, gerando menores limites de detecção e quantificação e, além disto, não são necessárias derivatizações para realização desta análise. No entanto, antes da análise cromatográfica, os analitos devem ser extraídos da matriz e concentrados em um solvente que seja compatível com os instrumentos analíticos. Não há método universal para extrair os analitos da matriz capilar. Este depende da estabilidade e da natureza química do composto em particular. ${ }^{106,107}$

Apesar dos problemas analíticos que técnicas de cromatografia líquida apresentam, estas são técnicas de escolha em análise de matrizes alternativas devido à sua alta sensibilidade, que é crucial considerando-se a baixa quantidade de amostra geralmente disponível nessas situações. ${ }^{108}$

Ainda, o cabelo fornece vantagens como facilidade na obtenção, coleta não invasiva, maior dificuldade para adulteração, janela de detecção ampla (Figura 8), além de raramente requerer cuidados no transporte e no armazenamento, o que favorece a estabilidade dos analitos. ${ }^{2,98,109}$ A verificação do uso de drogas de abuso por análise de cabelo é uma ferramenta útil para demonstrar ou excluir a suspeita de uso crônico. ${ }^{109,110}$

Foi relatado um método para quantificação de derivados e analgésicos opioides, utilizando CLAE/EM/EM. ${ }^{111}$ Um método de quan- 
tificação simultânea de opioides, anfetaminas, cocaína e metabólitos utilizando CG/EM em amostras de cabelo foi também relatado. ${ }^{12} \mathrm{Em}$ 2003, um estudo determinou canabinoides em cabelo após extração por headspace e EFS. ${ }^{113}$ Derivados anfetamínicos foram analisados em cabelo por CG/EM, ${ }^{114}$ CLAE/DF ${ }^{115}$ e CLAE/ESI-EM/EM após utilização de baixas doses de MDMA. ${ }^{116}$

\section{Unhas}

Nos últimos anos, a análise de fármacos em matrizes queratinizadas, como cabelo e unhas, tem recebido uma atenção considerável por causa de diversas vantagens sobre as metodologias de exames toxicológicos empregando fluidos biológicos, como urina ou soro. ${ }^{117}$ Assim como o cabelo, este tecido queratinizado pode armazenar fármacos administrados por meses, podendo fornecer evidências retrospectivas do uso de drogas de abuso. ${ }^{2}$

As unhas são compostas por uma proteína conhecida como queratina, que absorve as drogas durante o crescimento das unhas resultando em um período mais longo de detecção, bem como a impossibilidade de alteração dos testes. ${ }^{117}$ Porém, mais estudos são necessários para a correta interpretação dos dados obtidos.

\section{Suor}

Vários mecanismos de incorporação de medicamentos em suor têm sido sugeridos, incluindo a difusão passiva do sangue, passagem transdérmica de fármacos através da pele, dentre outros. Diariamente são produzidos entre 300 e $700 \mathrm{~mL}$ de suor, e 2-4 L/h podem ser produzidos durante exercício físico exaustivo. ${ }^{118}$

Em geral, as drogas são encontradas no suor, em vez de seus metabólitos polares, que normalmente predominam na urina. ${ }^{119}$ Contudo, a análise de drogas no suor raramente é realizada, porque é extremamente difícil estimar o volume de suor e coletar quantidades adequadas. Nos últimos anos, este empecilho parece ter sido resolvido pelo desenvolvimento de adesivos sweat patch, para coleta de suor.${ }^{118} \mathrm{O}$ suor também possui a vantagem de não representar coleta invasiva e de facilitar o controle de indivíduos internados em clínica de tratamento e detentos em liberdade condicional. ${ }^{97}$ Foi desenvolvido e validado um método para análise de derivados anfetamínicos com EFS, seguido de análise por CG/EM ${ }^{120}$ e por diagnóstico imunológico utilizando enzimaimunoensaio (ELISA). ${ }^{121}$

\section{Humor vítreo}

O material que preenche a cavidade posterior do olho potencialmente contém concentrações de drogas, apresentando boa correlação com a concentração correspondente no sangue. Além disso, o humor vítreo muitas vezes é a melhor amostra disponível em análises post-mortem como, por exemplo, em casos de corpos gravemente queimados ou decompostos.

A ausência de qualquer atividade metabólica significativa no olho sugere que as concentrações da droga no humor vítreo podem fornecer com precisão as concentrações da droga do corpo. ${ }^{91} \mathrm{O}$ MDMA e o MDEA, dentre outras designer drugs, podem ser determinados no humor vítreo e já possuem métodos validados. ${ }^{4}$

\section{Outras matrizes não convencionais}

Como afirmado anteriormente, o surgimento de métodos mais sensíveis e específicos permitiu testes para detecção de drogas em outras matrizes mais complexas e alternativas, com a finalidade de detecção precoce da exposição. Este é o caso da análise do tecido e sangue do cordão umbilical, e do líquido amniótico, que podem ser utilizados para documentar a exposição à droga no útero. ${ }^{108} \mathrm{~A}$ exposição pré-natal da droga é frequentemente determinada pelo relato materno ou pela análise toxicológica materna e/ou neonatal de fluidos biológicos e de tecidos. O mecônio é o primeiro material fecal do recém-nascido e é frequentemente analisado para determinar a exposição de drogas no período gestacional, devido à sua grande janela de detecção de drogas. Em comparação com testes neonatais de amostras de cabelo, a análise de mecônio é mais sensível para detectar a exposição in utero ao abuso de drogas. ${ }^{122} \mathrm{CG} / \mathrm{EM}$ e CLAE/EM foram utilizados para a detecção de aminas simpatomiméticas em mecônio, principalmente na análise de anfetaminas e metanfetaminas e, mais recentemente, MDMA, MDEA, MDA e MBDB. ${ }^{123}$ Existem outras matrizes alternativas, apesar de pouco utilizadas na rotina em análises toxicológicas, como é o caso do líquido pericárdico e do músculo esquelético, utilizados principalmente em situações post-mortem. ${ }^{124}$

\section{CONSIDERAÇÕES FINAIS}

Também conhecidos como "análogos", as designer drugs são drogas de abuso emergentes e estão entre as mais consumidas no mundo. A falta de conhecimento de dados farmacocinéticos e toxicológicos de grande parte destes compostos dificulta sua correta análise e identificação. Do ponto de vista analítico, a maioria destes compostos, principalmente os derivados mais novos, não está presente em bibliotecas espectrais comerciais e, portanto, pode representar desafios incomuns para laboratórios forenses. A identificação definitiva do tipo de droga geralmente requer uma análise aprofundada com a utilização de várias técnicas complementares, incluindo a espectroscopia no infravermelho, cromatografia em fase gasosa/espectrometria de massas e ressonância magnética nuclear. Exceto os derivados anfetamínicos, que já possuem métodos estabelecidos e confiáveis para triagem e confirmação, as outras classes de designer drugs precisam de mais estudos e aprimoramento de técnicas, métodos mais específicos e sensíveis, além de um tratamento da amostra mais eficaz. Além disto, cabe ressaltar que ao analisar amostras biológicas como, por exemplo, a urina, a maioria destes compostos é excretada sob forma de metabólitos, podendo estes ser moléculas de fármacos ou semelhantes aos metabólitos de drogas terapêuticas, como é o caso da $m \mathrm{CPP}$, que também é encontrada como metabólito do antidepressivo trazodona.

Embora estudos em humanos possibilitem a utilização de diferentes matrizes biológicas, urina e sangue são as de primeira escolha. No entanto, as designer drugs estão presentes no sangue e na urina (metabólitos) durante um curto período. Matrizes não convencionais, como o cabelo e as unhas, podem comprovar a utilização destas substâncias a longo prazo. Além disto, o uso de amostras alternativas está ganhando importância no campo da toxicologia, devido a seus procedimentos de coletas não invasivas. Os equipamentos analíticos tornam-se cada vez mais sensíveis e específicos, permitindo a detecção e quantificação de drogas em quantidades muito baixas. $\mathrm{O}$ cabelo e o fluido oral têm sido as amostras não convencionais mais amplamente utilizadas, principalmente em situações forenses.

Por fim, o objetivo principal desta revisão foi realizar uma pesquisa a respeito das principais designer drugs encontradas no mercado ilícito, assim como dar um panorama dos principais métodos desenvolvidos para análise e respectivas matrizes biológicas convencionais e não convencionais, como uma forma de orientar o analista na busca por um resultado fidedigno, com a qualidade que uma análise para fins forenses requer. Contribuindo, assim, para que profissionais e estudantes obtenham informações atuais sobre os métodos validados em diferentes matrizes biológicas para determinação de designer drugs, que estão sendo encontradas cada vez mais no país.

\section{REFERÊNCIAS}

1. Gahlinger, P.; Illegal Drugs: A Complete Guide to Their History, Chemistry, Use and Abuse, $1^{\text {st }}$ ed., Sagebrush: Salt Lake City, 2001. 
2. Costa, J. L.; Dissertação de Mestrado, Universidade de São Paulo, Brasil, 2004.

3. Henderson, G. L.; J. Forensic Sci. 1988, 33, 569.

4. De Letter, E. A.; Clauwaert, K. M.; Lambert, W. E.; van Bocxlaer, J. F.; De Leenheer, A. P.; Piette, M. H.; J. Anal. Toxicol. 2002, 26, 113.

5. Theobald, D. S.; Fehn, S.; Maurer, H. H.; J. Mass Spectrom. 2005, 40, 105.

6. De Boer, D.; Bosman, I. J.; Hidvegi, E.; Manzoni, C.; Benko, A. A.; Dos Reys, L. J.; Forensic Sci. Int. 2001, 121, 47.

7. Gilman, A. G.; Goodman \& Gilman's: the Pharmacological Basis of Therapeutics, 10 $0^{\text {th }}$ ed., McGraw-Hill: New York, 2001.

8. Moro, E. T.; Ferraz, A. A. F.; Modolo, N. S. P.; Rev. Bras. Anestesiol. 2006, 56,2 .

9. Costa, J. L.; Chasin, A. A. M.; J. Chromatogr., B: Anal. Technol. Biomed. Life Sci. 2004, 811, 41.

10. Peters, F. T.; Martinez-Ramirez, J. A.; Ther. Drug Monit. 2010, 32, 532.

11. Buchanan, J. F.; Brown, C. R.; Med. Toxicol. Adverse Drug Exp. 1988, 3,1 .

12. Takahashi, M.; Nagashima, M.; Suzuki, J.; Seto, T.; Yasuda, I.; Yoshida, T.; J. Health Sci. 2008, 54, 89.

13. Peters, F. T.; Schaefer, S.; Staack, R. F.; Kraemer, T.; Maurer, H. H.; J. Mass Spectrom. 2003, 38, 659.

14. Staack, R. F.; Maurer, H. H.; Curr. Drug Metab. 2005, 6, 259.

15. Nagai, F.; Nonaka, R.; Hisashi, S.; Kamimura, K.; Eur. J. Pharmacol. 2007, 559, 132

16. DEA, United States; Schedules of controlled substances: temporary placement of alphamethyltryptamine and 5-methoxy- $N, N$-diisopropyltryptamine into Schedule I, Department of Justice, Drug Enforcement Administration (DEA), Final rule, Fed. Regist. 68, 16427, 2003.

17. DEA, United States; Schedules of controlled substances: placement of alpha-methyltryptamine and 5-methoxy- $N, N$-diisopropyltryptamine into schedule I of the Controlled Substances Act, Department of Justice, Drug Enforcement Administration (DEA), Final rule, Fed. Regist. 69, 58950, 2004.

18. Uchiyama, N.; Kikura-Hanajiri, R.; Ogata, J.; Goda, Y.; Forensic Sci. Int. 2010, 198, 31.

19. Parrott, A.; Morinan, A.; Moss, M.; Scholey, A.; Understanding Drugs and Behaviour, Wiley \& Sons: West Sussex, 2004.

20. Green, A. R.; Mechan, A. O.; Elliott, J. M.; O'shea, E.; Colado, M. I.; Pharmacol. Rev. 2003, 55, 463.

21. Nichols, D. E.; J. Psychoactive Drugs 1986, 18, 305.

22. Shulgin, A. P.; A Chemical Love Story, $1^{\text {st }}$ ed., Transform Press: Berkley, 1991.

23. Fahn, S.; New Engl. J. Med. 1996, 335, 2002.

24. Henderson, G.; J. Forensic Sci. 1991, 36, 422.

25. Davis, G. C.; Williams, A. C.; Markey, S. P.; Ebert, M. H.; Caine, E. D.; Reichert, C. M.; Kopin, I. J.; Psychiat. Res. 1979, 1, 249.

26. Springer, D.; Fritschi, G.; Maurer, H. H.; J. Chromatogr., B: Anal. Technol. Biomed. Life Sci. 2003, 796, 253.

27. Higashikawa, Y.; Suzuki, S.; Forensic Toxicol. 2008, 26, 1.

28. Skulska, A.; Kala, M.; Parczewski, A.; Przegl Lek. 2005, 62, 581.

29. Langston, W. Em Parkinson's Disease. Diagnosis and Clinical Management; Factor, S. A.; Weiner, W. J., eds.; Demos Medical Publishing: New York, 2002.

30. Barone, J. A.; Shermock, K. M.; J. Pharm. Pract. 1997, 10, 292.

31. Oga, S.; Camargo, M. M. A.; Batistuzzo, J. A. O.; Fundamentos de Toxicologia, $3^{a}$ ed., Editora Atheneu: São Paulo, 2008.

32. Schep, L. J.; Slaughter, R. J.; Beasley, D. M.; Clin. Toxicol. 2010, 48, 675.

33. Klaassen, C. D.; Casarett and Doull's Toxicology: the basic science of poisons, $6^{\text {th }}$ ed., McGraw-Hill: New York, 2001.

34. Johansen, S. S.; Hansen, A. C.; Mueller, I. B.; Lundemose, J. B.; Franzmann, M. B.; J. Anal. Toxicol. 2003, 27, 253.
35. Felscher, D.; Schulz, K.; J. Forensic Sci. 2000, 45, 1327.

36. Ewald, A. H.; J. Chromatogr, B: Anal. Technol. Biomed. Life Sci. 2005, $824,123$.

37. Theobald, D. S.; Maurer, H. H.; Biochem. Pharmacol. 2007, 73, 287.

38. De Boer, D.; Bosman, I.; Pharm. World Sci. 2004, 26, 110.

39. Kanai, K.; Takekawa, K.; Kumamoto, T.; Ishikawa, T.; Ohmori, T.; Forensic Toxicol. 2008, 26, 6.

40. Carmo, H.; Boer, D.; Remião, F.; Carvalho, F.; Reys, L. A.; Bastos, M. L.; J. Chromatogr., B: Anal. Technol. Biomed. Life Sci. 2004, 811, 143.

41. Carmo, H.; Hengstler, J. G.; Boer, D.; Ringel, M.; Remiao, F.; Carvalho, F.; Fernandes, E.; Reys, L. A.; Oesch, F.; Bastos, M. D.; Toxicology 2005, 206, 75 .

42. http://www.nida.nih.gov/researchReports/ecstasy/ecstasy.html, acessada em Maio 2009.

43. Giroud, C.; Augsburger, M.; Rivier, L.; Mangin, P.; Sadeghipour, F.; Varesio, E.; Veuthey, J. L.; Kamalaprija, P.; J. Anal. Toxicol. 1998, 22, 345.

44. Cole, M. D.; Lea, C.; Oxley, N.; Sci. Justice 2002, 42, 223.

45. Boatto, G.; Nieddu, M.; Carta, A.; Pau, A.; Palomba, M.; Asproni, B.; Cerri, R.; J. Chromatogr, B: Anal. Technol. Biomed. Life Sci. 2005, 814, 93.

46. Curtis, B.; Kemp, P.; Harty, L.; Choi, C.; Christensen, D.; J. Anal. Toxicol. 2003, 27, 493.

47. Habrdova, V.; Peters, F. T.; Theobald, D. S.; Maurer, H. H.; J. Mass Spectrom. 2005, 40, 785.

48. Theobald, D. S.; Putz, M.; Schneider, E.; Maurer, H. H.; J. Mass Spectrom. 2006, 41, 872.

49. Lood, Y.; Eklund, A. Em Abstract book of the $43^{\text {rd }}$ International SOFT/ TIAFT Meeting in Washington; LeBeau, M., ed.; TIAFT2004: Washington, 2004, p. 115

50. De Boer, D.; Egberts, T.; Maes, R. A. A.; Pharm. World Sci. 1999, 21, 47.

51. Theobald, D. S.; Fritschi, G.; Maurer, H. H.; J. Chromatogr., B: Anal. Technol. Biomed. Life Sci. 2006, 842, 76.

52. Kanamori, T.; Inoue, H.; Iwata, Y.; Ohmae, Y.; Kishi, T.; J. Anal. Toxicol. 2002, 26, 61

53. De Boer, D.; Lesseps, J.; Gijzels, M.; Pilon, N.; Bosman, I. J.; Maes, R. A. A.; Proceedings of the 1998 Joint SOFT/TIAFT Meeting, $5^{\text {th }}$ ed., TIAFT98: Albuquerque, 1999

54. Lin, L. C.; Liu, J. T.; Chou, S. H.; Lin, C. H.; J. Chromatogr., B: Anal. Technol. Biomed. Life Sci. 2003, 798, 241.

55. Kikura-Hanajiri, R.; Hayashi, M.; Saisho, K.; Goda, Y.; J. Chromatogr., B: Anal. Technol. Biomed. Life Sci. 2005, 825, 29.

56. Kovar, K. A.; Pharmacopsychiatry 1998, 2, 69

57. Glennon, R. A.; Young, R.; Rosecrans, J. A.; Anderson, G. M.; Biol. Psychiatry 1982, 17, 807.

58. Halberstadt, A. L.; Geyer, M. A.; Neuropharmacology 2011, 61, 364.

59. Cunningham, N.; Emerg. Med. Australas 2008, 20, 167.

60. Riba, J.; Rodríguez-Fornells, A.; Urbano, G.; Morte, A.; Antonijoan, R.; Montero, M.; Callaway, J. C.; Barbanoj, M. J.; Psychopharmacology 2001, 154, 85.

61. Meatherall, R.; Sharma, P.; J. Anal. Toxicol. 2003, 27, 313.

62. Mccandless, D.; The Guardian (Electronic Version), 2004

63. Takahashi, M.; Nagashima, M.; Suzuki, J.; Seto, T.; Yasuda, I.; Yoshida, T.; J. Health Sci. 2008, 54, 89.

64. Wikström, M.; Holmgren, P.; Ahlner, J.; J. Anal. Toxicol. 2004, 28, 67.

65. Bauman, M. H.; Clark, R. D.; Budzynski, A. G.; Partilla, J. S.; Blough, B. E.; Rothman, R. B.; Ann. N. Y. Acad. Sci. 2004, 1025, 189.

66. Bauman, M. H.; Clark, R. D.; Budzynski, A. G.; Partilla, J. S.; Blough, B. E.; Rothman, R. B.; Neuropsychopharmacology 2005, 30, 550.

67. http://www.aspecgo.com.br/artigos/e760ebcb10014859938a4edc2021eacd.pdf, acessada em Agosto 2011

68. Stanaszek, R.; Zuba, D.; Problems Forensic Sci. 2006, 66, 220. 
69. Lanaro, R.; Costa, J. L.; Zanolli Filho, L. A.; Cazenave, S. O. S.; Quim. Nova 2010, 33, 725.

70. Maurer, H. H.; Microgram. J. 2004, 2, 22.

71. http://e-legis.anvisa.gov.br/leisref/public/showAct.php?id= 33987\&word=, acessada em Agosto 2011.

72. Uchiyama, N.; Kikura-Hanajiri, R.; Kawahara, N.; Haishima, Y.; Goda, Y.; Chem. Pharm. Bull. 2009, 27, 439.

73. Müller, H.; Sperling, W.; Köhrmann, M.; Huttner, H.; Kornhuber, J.; Maler, J.; Schizophr. Res. 2010, 118, 309.

74. Auwärter, V.; Dresen, S.; Weinmann, W.; Muller, M.; Putz, M.; Ferreiros, N.; J Mass Spectrom. 2009, 44, 832.

75. Sobolevsky, T.; Prasolov, I.; Rodchenkov, G.; Forensic Sci. Int. 2010, 200, 141.

76. Moreau, R. L. De M.; Siqueira, M. E. P. B.; Ciências Farmacêuticas: Toxicologia Analítica, Editora Guanabara Koogan: Rio de Janeiro, 2008.

77. Drummer, O. H.; Anal. Bioanal. Chem. 2007, 388, 1495.

78. Namera, A.; Nakamoto, A.; Saito, T.; Nagao, M.; Forensic Toxicol. 2011, $29,1$.

79. Rodriguez-Cruz, S. E.; Rapid Commun. Mass Spectrom. 2006, $20,53$.

80. Causon, R.; J. Chromatogr., B: Biomed. Sci. Appl. 1997, 689, 175.

81. Chasin, A. A. M.; Rev. Bras. Toxicol. 2001, 14, 15.

82. Gentili, S.; Cornetta, M.; Macchia, T.; J. Chromatogr, B: Anal. Technol. Biomed. Life Sci. 2004, 801, 289.

83. Battu, C.; Marquet, P.; Fauconnet, A. L.; Lacassie, E.; Lachatre, G.; J. Chromatogr. Sci. 1998, 36, 1.

84. Pellegrini, M.; Rosati, F.; Pacifici, R.; Zuccaro, R.; Romolo, F. S.; Lopez, A.; J. Chromatogr., B: Anal. Technol. Biomed. Life Sci. 2002, 769, 243.

85. Stout, P. R.; Klette, K. L.; Horn, C. K.; J. Forensic Sci. 2004, 49, 160.

86. Queiroz, M. E. C.; Lanças, F. M.; Quim. Nova 2005, $28,5$.

87. Chasin, A. A. M.; Dissertação de Mestrado, Universidade de São Paulo, Brasil, 1990.

88. Chasin, A. A. M.; Tese de Doutorado, Universidade de São Paulo, Brasil, 1996.

89. Joseph, R. E.; Oyler, J. M.; Wstadik, A. T.; Ohuoha, C.; Cone, E. J.; Pharmacol. J. Anal. Toxicol. 1998, 22, 6.

90. Staub, C.; J. Chromatogr. 1999, 733, 119.

91. Clauwaert, K. M.; van Bocxlaer, J. F.; De Letter, E. A.; van Calenbergh, S.; Lambert, W. E.; De Leenheer, A. P.; Clin. Chem. 2000, 46, 1968.

92. Blanke, R. V.; Poklis, A. Em Casarett and Doull's toxicology: the basic science of poisons; Klaassen, C. D.; Amdur, M. O.; Doull, J., eds.; McGrall-Hill: New York, 1996, p. 905.

93. Wohlfarth, A.; Weinmann, W.; Dresen, S.; Anal. Bioanal. Chem. 2010, 396, 2403.

94. Clauwaert, K. M.; Bocxlaer, J. F. V.; Leenheer, A. P.; Forensic Sci. Int. 2001, 124, 36.

95. Drummer, O.H.; Gerostamoulos, J.; Ther. Drug Monit. 2002, 24, 199.

96. http://www.obid.senad.gov.br/portais/OBID/biblioteca/documentos/ Publicacoes/328 033.pdf, acessada em Agosto 2011.

97. Kintz, P.; Samyn, N.; J. Chromatogr. B: Biomed. Sci. Appl. 1999, 733, 137.

98. Fucci, N.; Giovanni, N.; Chiarotti, M.; Forensic Sci. Int. 2003, 134, 40.

99. Navarro, M.; Pichini, S.; Farre, M.; Ortuno, J.; Roset, P. N.; Segura, J.; De La Torre, R.; Clin. Chem. 2001, 47, 1788.

100. Samyn, N.; De Boeck, G.; Wood, M.; Lamers, C. T.; De Waard, D.; Brookhuis, K. A.; Verstraete, A. G.; Riedel, W. J.; Forensic Sci. Int. 2002, 128, 90.

101. Mortier, K. A.; Maudens, K. E.; Lambert, W. E.; Clauwaert, K. M.; Van Bocxlaer, J. F.; Deforce, D. L.; Van Peteghem, C. H.; De Leenheer, A. P.; J. Chromatogr., B: Anal. Technol. Biomed. Life Sci. 2002, 779, 321.
102. Samyn, N.; Laloup, M.; De Boeck, G.; Anal. Bioanal. Chem. 2007, 388, 1437.

103. Concheiro, M.; de Casto, A.; Quintela, O.; Lopez-Rivadulla, M.; Cruz, A.; Forensic Sci. Int. 2005, 150, 221.

104. Scheidweiler, K. B.; Huestis, M. A.; J. Chromatogr., B: Anal. Technol. Biomed. Life Sci. 2006, 835, 90.

105. Peters, F. T.; Samyn, N.; Kraemer, T.; Riedel, W. J.; Maurer, H. H.; Clin. Chem. 2007, 53, 702.

106. Girod, C.; Staub, C.; Forensic Sci. Int. 2000, 107, 261.

107. Tsanaclis, L.; Wicks, J. F. C.; Forensic Sci. Int. 2008, 176, 19.

108. Gallardo, E.; Queiroz, J. A.; Biomed. Chromatogr. 2008, $22,795$.

109. Rothe, M.; Pragst, F.; Spiegel, K.; Harrach, T.; Fischer, K.; Kunkel, J.; Forensic Sci. Int. 1997, 89, 111.

110. Moore, C.; Marinetti, L.; Coulter, C.; Crompton, K.; Forensic Sci. Int. 2008, 176, 47.

111. Musshoff, F.; Lachenmeier, K.; Trafkowski, J.; Madea, B.; Nauck, F.; Stamer, U.; Ther. Drug Monit. 2007, 29, 655.

112. Cordero, P.; J. Chromatogr., B: Anal. Technol. Biomed. Life Sci. 2007, $850,423$.

113. Musshoff, F.; Lachenmeier, D. W.; Kroener, L.; Madea, B.; Forensic Sci. Int. 2003, 133, 32.

114. Villamor, J. L.; Bermejo, A. M.; Fernández, P.; Tabernero, M. J.; J. Anal. Toxicol. 2005, 29, 135.

115. Nakamura, S.; Tomita, M.; Wada, M.; Chung, H.; Kuroda, N.; Nakashima, K.; Biomed. Chromatogr. 2006, 20, 622.

116. Chèze, M.; Deveaux, M.; Martin, C.; Lhermitte, M.; Pépin, G.; Forensic Sci. Int. 2007, 170, 100.

117. Engelhart, D. A.; Lavins, E. S.; Sutheimer, C. A.; J. Anal. Toxicol. 1998, $22,314$.

118. Kintz, P.; Samyn, N.; Handbook of Analytical Separations, Editora Elsevier Science: Amsterdam, 2000.

119. Follador, M. J. D.; Yonamine, M.; Silva, R. L.; J. Chromatogr., B: Anal. Technol. Biomed. Life Sci. 2004, 811, 37.

120. De Martinis, B. S.; Barnes, A. J.; Scheidweiler, K. B.; Huestis, M. A.; J. Chromatogr., B: Anal. Technol. Biomed. Life Sci. 2007, 852, 450.

121. Pichini, S.; Navarro, M.; Pacifici, R.; Zuccaro, P.; Ortuño, J.; Farré, M.; Roset, P.; Segura, J.; de la Torre, R.; J. Anal. Toxicol. 2003, 27, 294.

122. Gray, T.; Huestis, M.; Anal. Bioanal. Chem. 2007, 388, 1455.

123. Pichini, S.; Pacifici, R.; Pellegrini, M.; Anal. Chem. 2004, 76, 2124.

124. Moriya, F.; Hashimoto, Y.; Legal Med. 1999, 1, 86.

125. Pichini, S.; Pujadas, M.; Marchei, E.; Pellegrini, M.; Fiz, J.; Pacifici, R.; Zuccaro, P.; Farre, M.; de la Torre, R.; J. Pharm. Biomed. Anal. 2008, 47, 335.

126. Ewald, A. H.; Peters, F. T.; Weise, M.; Maurer, H. H.; J. Chromatogr., B: Anal. Technol. Biomed. Life Sci. 2005, 824, 123.

127. Ewald, A. H.; Puetz, M.; Maurer, H. H.; J. Chromatogr, B: Anal. Technol. Biomed. Life Sci. 2008, 862, 252.

128. Ewald, A. H.; Fritschi, G.; Bork, W. R.; Maurer, H. H.; J. Mass Spectrom. 2006, 41, 487.

129. Staack, R. F.; Fehn, J.; Maurer, H. H.; J. Chromatogr., B: Anal. Technol. Biomed. Life Sci. 2003, 789, 27.

130. Staack, R. F.; Fritschi, G.; Maurer, H. H.; J. Chromatogr., B: Anal. Technol. Biomed. Life Sci. 2002, 773, 35.

131. Staack, R. F.; Fritschi, G.; Maurer, H. H.; J. Mass Spectrom. 2003, 38, 971.

132. Maurer, H. H.; Kraemer, T.; Springer, D.; Staack, R. F.; Ther. Drug Monit. 2004, 26, 127.

133. Moore, C.; Rana, S.; Coulter, C.; J. Chromatogr., B: Anal. Technol. Biomed. Life Sci. 2007, 852, 459.

134. Lucas, N.; Dissertação de Mestrado, Universidade de Waikato, Nova Zelândia, 2008. 